\title{
Testing of Multi-Axial Strength Behavior of Hard Foams
}

\author{
V. A. Kolupaev ${ }^{1, a}$, A. Bolchoun ${ }^{1}$, and H. Altenbach ${ }^{2}$ \\ 1 German Institute for Polymers (DKI), Schlossgartenstrasse 6, D-64289 Darmstadt \\ 2 Martin Luther University Halle-Wittenberg, Kurt-Mothes-Str.1, D-06099 Halle (Saale)
}

\section{Summary}

For modern applications of hard foams an appropriate description of the limit behavior under different stress states are required. So a new strength hypothesis based on the stress-angle is proposed. In order to obtain the parameters of the model, relevant tests are discussed.

The quality of approximation of the experimental data by a limit surface must be evaluated. Some evaluation criteria are discussed and applied to the measured data from the literature. The results of the fitting are presented in the BURZYŃSKI-plane and in the $\pi$-plane. The presented approach reduces the number of errors in fitting of the limit surface. Certain empiric restrictions help to obtain reliable fitting even for insufficient or unsure measurements.

\section{Experiments}

An upgraded tensile testing machine ZWICK 1382 is used for tension and compression tests (Figs 1 and 2). The machine is equipped with a torsion drive, which besides torsion itself allows to apply combined loadings such as tension or compression combined with torsion. The tension, compression, and torsion tests alone do not yield a sufficient amount of information about the limit behavior of hard foams. Especially 3D-generalizations using a strength hypothesis lead to uncertainties. In order to increase the reliability in description of the material behavior additional tests are discussed.

The enlacement test (Fig. 3) can be carried out relatively easy. It is used to implement uniform 2D-compression. In the intersection a foil is placed, which is necessary to achieve a homogeneous distribution of the stress. The edges of the specimen are treated in order to reduce the friction. The experiment can be carried out with a common tensile testing machine. An inner pressure test with a tubular specimen can be carried out (Fig. 4). A biaxial tension test can be carried out with a conventional tensile test machine (Fig. 5).

The 3D tests are very important. Triaxial compression (hydrostatic compression) is carried out in a pressure chamber. The scheme (Fig. 7) is used to implement an approximately uniform triaxial tension. In this case the stress concentration occurs at the corners, where the cuts of the "maltese" cube are met [2]. Those regions with stress concentrators can be treated, however this does not solve the problem of inhomogeneous stress distribution. Together with the test in a pressure chamber, those six tests provide a good basis for description of the limit surface.

\footnotetext{
a e-mail: VKolupaev@dki.tu-darmstadt.de
} 


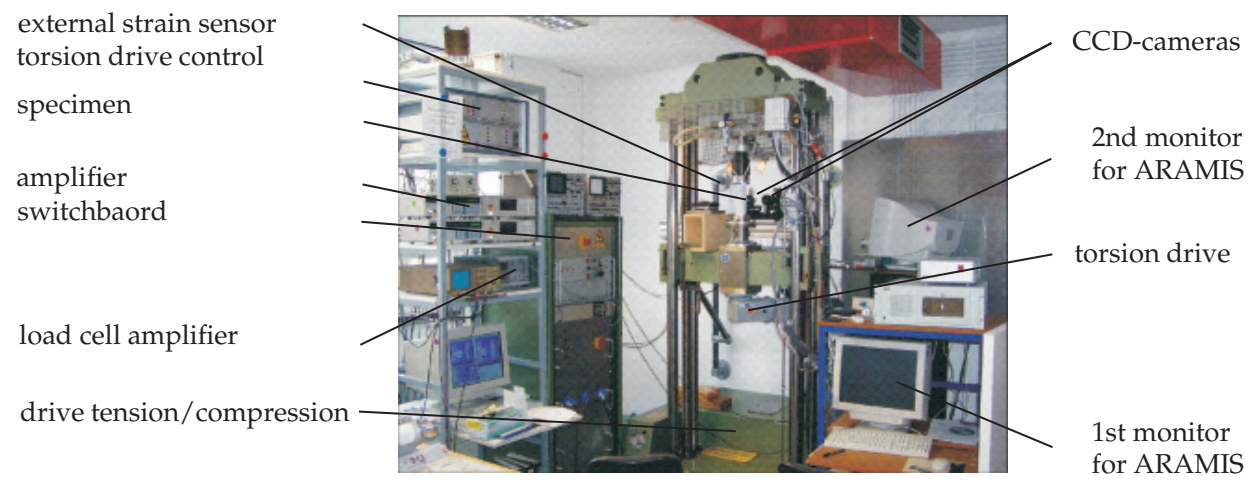

Fig. 1. Experimental equipment (left: switchboard, right: upgraded testing machine ZWICK 1382 with measuring system ARAMIS).

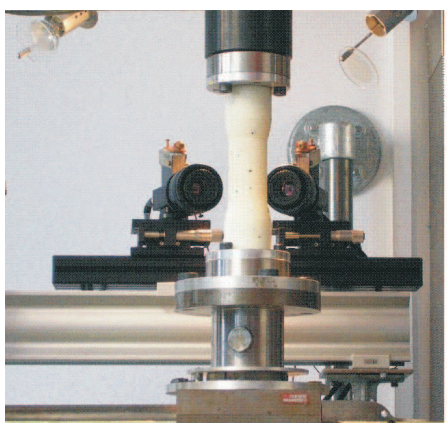

front view

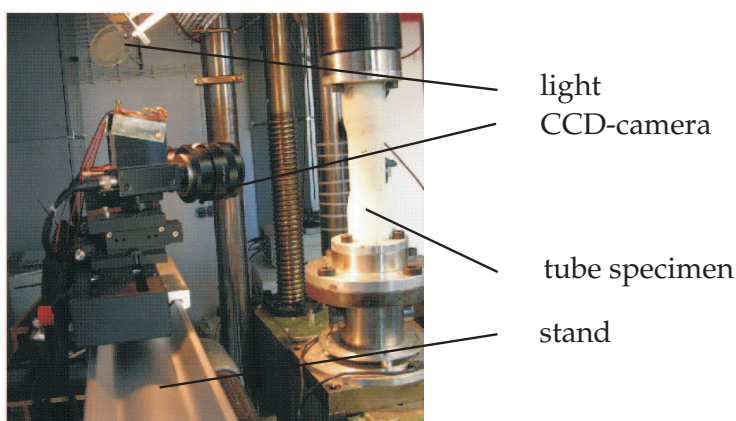

side view

Fig. 2. Displacement recording with two CCD-cameras Sony XC75-CE (8bit b/w) for ARAMIS, gom, maximal angle $60^{\circ}$

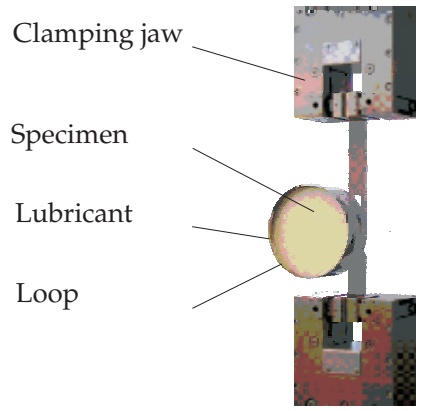

Fig. 3. Biaxial compression (enlacement) test of a foam disc made of Rohacell-WF-51 with the diameter $\varnothing 120 \mathrm{~mm}$ and thickness $32 \mathrm{~mm}$, the loop is made of stainless steel $18 \mathrm{Cr} 9 \mathrm{Ni}$ with $0,2 \mathrm{~mm}$ thickness.

\section{Model and Fitting to the Experimental Results}

The strength hypothesis based on the stress angle $\theta$ with $\cos 3 \theta=3 \sqrt{3} I_{3}^{\prime} /\left(2\left(I_{2}^{\prime}\right)^{3 / 2}\right)$ as a function of the deviatoric stress invariants $I_{2}^{\prime}=\frac{1}{2} s \cdot \cdot s, I_{3}^{\prime}=\frac{1}{3}(s \cdot s) \cdot s$ and $I_{1}=I \cdot \cdot \sigma$ has two forms [3-7]

$$
\left(3 I_{2}^{\prime}\right)^{3} \frac{1+c_{3} \cos 3 \theta+c_{6} \cos ^{2} 3 \theta}{1+c_{3}+c_{6}}=\left(\frac{\sigma_{\mathrm{eq}}-\gamma_{1} I_{1}}{1-\gamma_{1}} \frac{\sigma_{\mathrm{eq}}-\gamma_{2} I_{1}}{1-\gamma_{2}}\right)^{3},
$$



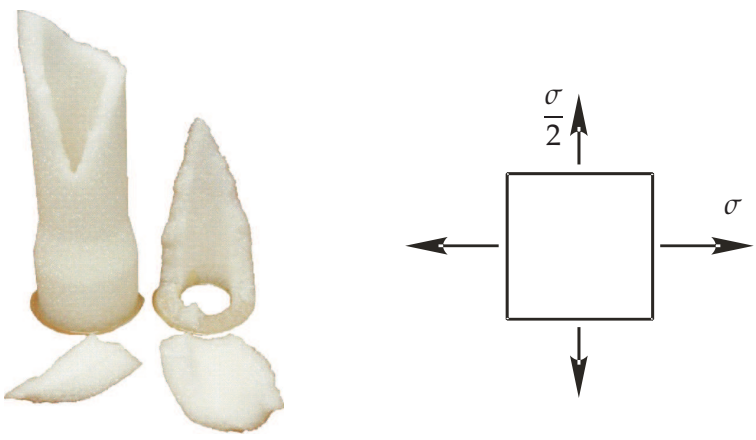

Fig. 4. Inner pressure test using a tube specimen with dick walls made of Rohacell $51 \mathrm{WF}: \varnothing_{\min }=30$, $\varnothing_{\max }=48$, length of the parallel part $L=80 \mathrm{~mm}$ (with distilled water as pressure medium)

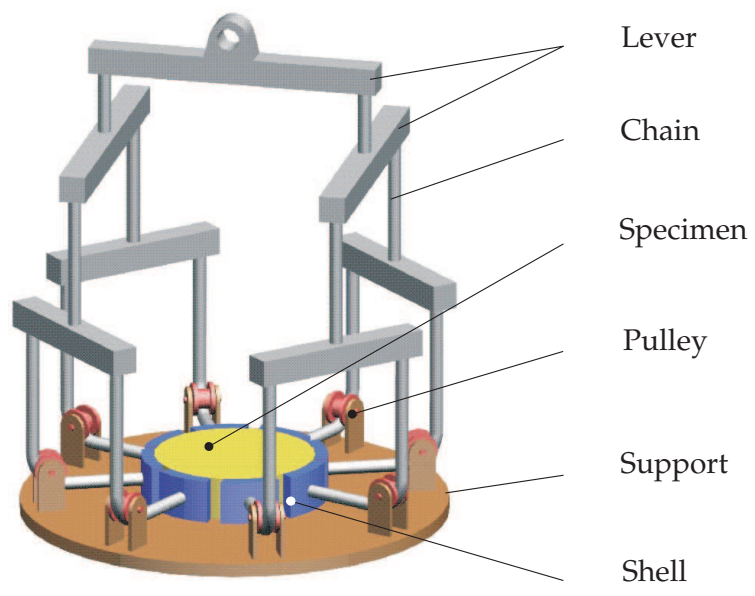

Fig. 5. A device for biaxial tension test for hard foams (specimen is a disc with diameter $\varnothing 120 \mathrm{~mm}$ and thickness Dicke $32 \mathrm{~mm}$ )

$$
\left(3 I_{2}^{\prime}\right)^{3} \frac{1+c_{3} \cos 3 \theta+c_{6} \cos ^{2} 3 \theta}{1+c_{3}+c_{6}}=\left(\frac{\sigma_{\mathrm{eq}}-\gamma_{1} I_{1}}{1-\gamma_{1}} \frac{\sigma_{\mathrm{eq}}-\gamma_{2} I_{1}}{1-\gamma_{2}} \frac{\sigma_{\mathrm{eq}}-\gamma_{3} I_{1}}{1-\gamma_{3}}\right)^{2} .
$$

The hydrostatic nodes are defined in (1) using $\gamma_{1} \in\left[0 ; 1\left[\right.\right.$ and $\gamma_{2}<0$, while for the second model $\gamma_{1}=\gamma_{2} \in\left[0 ; 1\left[, \gamma_{3}<0 ; \gamma_{1} \in\left[0 ; 1\left[, \gamma_{2}=\gamma_{3}<0\right.\right.\right.\right.$ or $\gamma_{1} \in\left[0 ; 1\left[, \gamma_{2}<0, \gamma_{3}=0\right.\right.$. The parameters $c_{3}$ and $c_{6}$ in (1) and (2) are bounded by (Fig. 8):

$$
c_{6}=\frac{1}{4}\left(2+c_{3}\right), \quad c_{6}=\frac{1}{4}\left(2-c_{3}\right), \quad c_{6}=\frac{5}{12} c_{3}^{2}-\frac{1}{3} .
$$

Setup $c_{3}=0$ yields surfaces with hexagonal symmetry. With $c_{3}=c_{6}=0$ rotationally symmetric models are obtained. The model contains a number of well-known models or is able to approximate them $[2,3]$. The parameters of the models are computed from the measured data.

If the associated flow rule is used to compute the POISSON's ratio at tension and compression, it yields the useful restrictions $\left.\left.v_{+}^{\mathrm{pl}} \in\right]-1 ; 1 / 2\right]$ and $\left.\left.v_{-}^{\mathrm{pl}} \in\right]-1 ; 1 / 2\right]$ for the parameters of the model. It follows for Eq. (1) and Eq. (2), respectively:

$$
\begin{gathered}
v_{+}^{\mathrm{pl}}=\frac{-1+2\left(\gamma_{1}+\gamma_{2}\right)-3 \gamma_{1} \gamma_{2}}{-2+\gamma_{1}+\gamma_{2}}, \\
v_{+}^{\mathrm{pl}}=\frac{3-5\left(\gamma_{1}+\gamma_{2}+\gamma_{3}\right)+7\left(\gamma_{1} \gamma_{2}+\gamma_{1} \gamma_{3}+\gamma_{2} \gamma_{3}\right)-9 \gamma_{1} \gamma_{2} \gamma_{3}}{6-4\left(\gamma_{1}+\gamma_{2}+\gamma_{3}\right)+2\left(\gamma_{1} \gamma_{2}+\gamma_{1} \gamma_{3}+\gamma_{2} \gamma_{3}\right)} .
\end{gathered}
$$




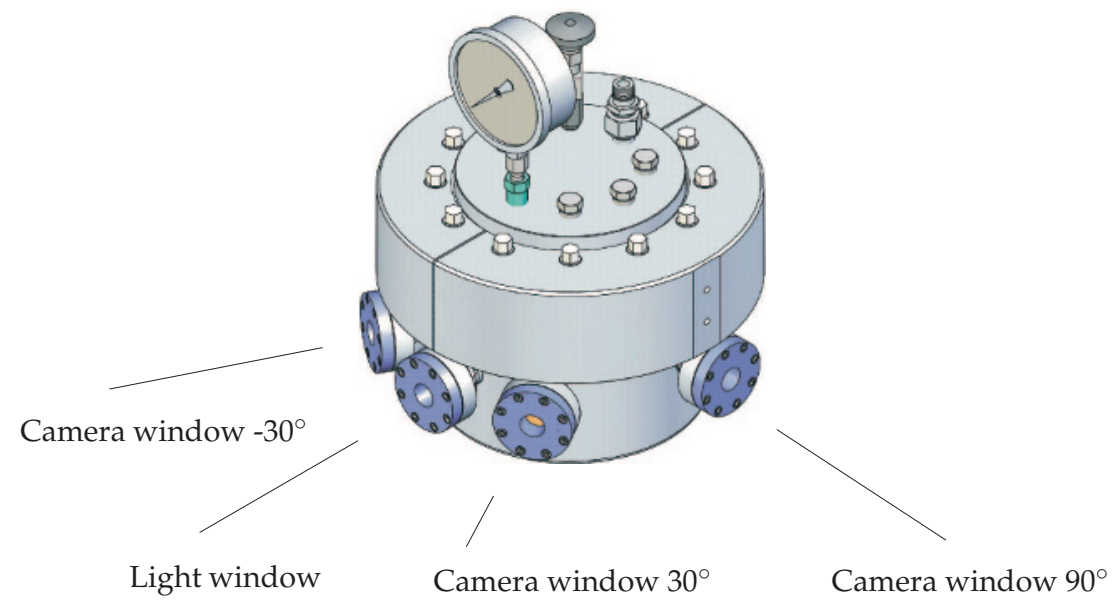

Fig. 6. Triaxial compression loading in the pressure chamber by PARR Instrument CO., Moline. The maximal pressure is $150 \mathrm{bar}$, Pressure medium - distilled water, specimen geometry - cube $10 \times 10 \times$ $10 \mathrm{~mm}$

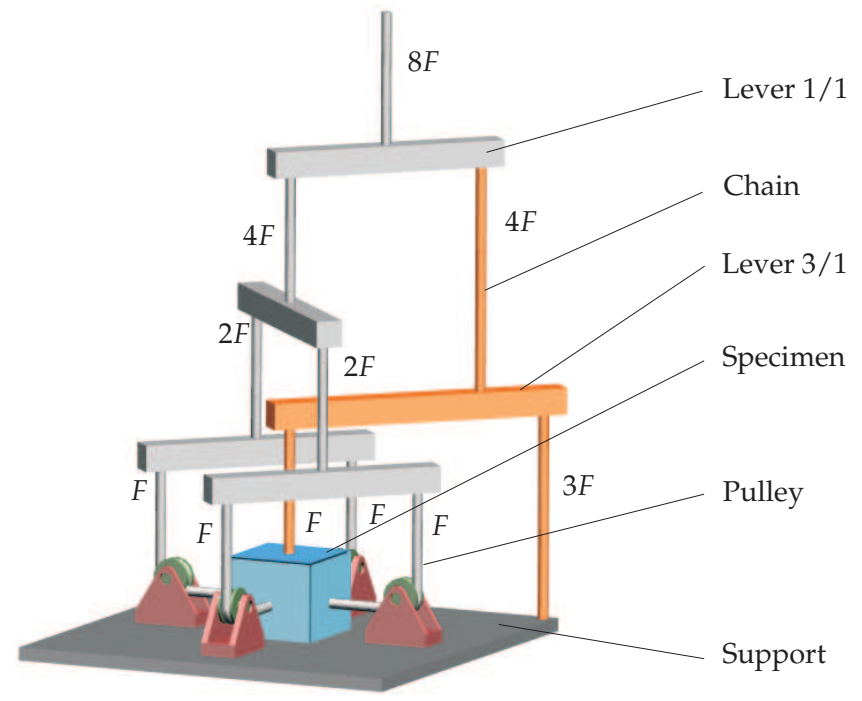

Fig. 7. Scheme of a device for hydrostatic tension test for hard foams with the "maltese" cube specimen of DESHPANDE. The notations $1 / 1$ and $3 / 1$ denote the arm ratio of the lever. $F$ shows the force distribution in the chains. Problems: weight of the links, non-coaxial application of forces and change of angle, when the lateral acting forces are applied.

The expressions for $v_{-}^{\mathrm{pl}}$ are generally rather large. The parameters $c_{3}, c_{6}$ and $\gamma_{i}$ can be obtained using an optimization routine.

\section{Objective Functions for Evaluation of 3D-Failure}

Let us consider a failure surface, which is given in the form $\Phi\left(\sigma_{\mathrm{I}}, \sigma_{\mathrm{II}}, \sigma_{\mathrm{III}}\right)=0$ and a set of experimental data in the principal stress space $\left(\sigma^{i}, \tau^{i}\right), i=1 \ldots n$. The quality of approximation of the experimental data by the surface must be evaluated. Four evaluation criteria are discussed (Fig. 9). These criteria cannot be used to estimate the parameters of the model be- 


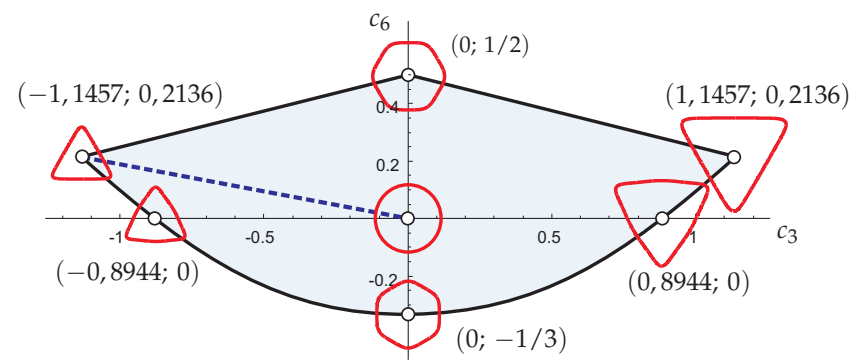

Fig. 8. The region in the parameter space $c_{3}-c_{6}$, which ensures convexity of the geomechanical model $\left(\gamma_{i}=0\right)$ in the $\pi$-plane

cause of their computational complexity. However, they can be applied to compare different sets of model parameters.

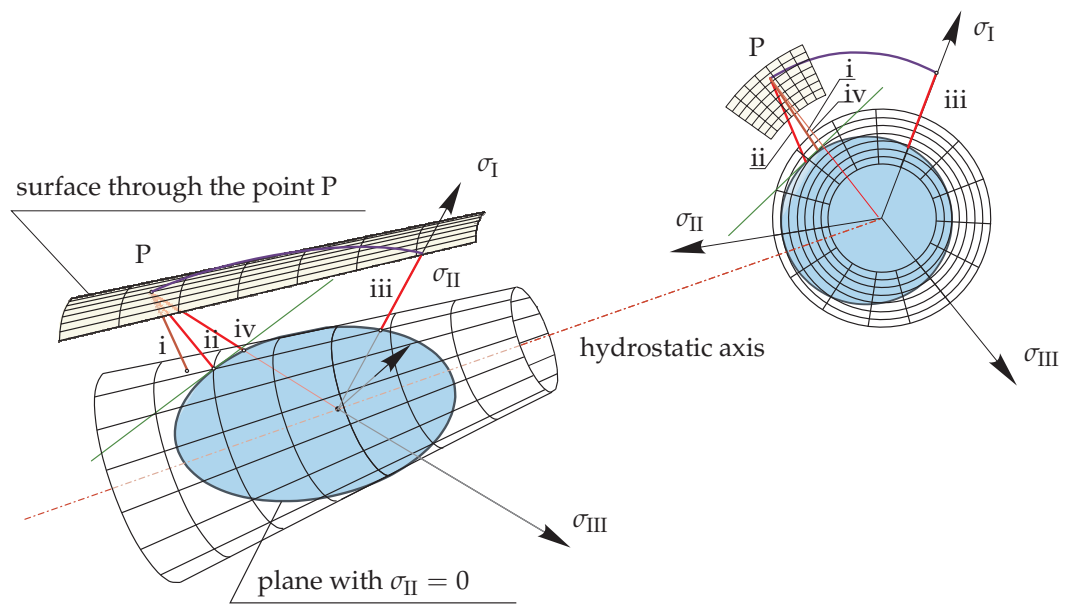

Fig. 9. Cone potential in the principal stress space. Comparing of four evaluation criteria.

i) The quality of regression in the principal stress space is evaluated. For every measurement the distance to the failure surface in the principal stress space $\left(\sigma_{\mathrm{I}}, \sigma_{\mathrm{II}}, \sigma_{\mathrm{III}}\right)$ is computed and then averaged over all measurements. That means, that for each measurement $\left(\sigma_{\mathrm{I}}^{i}, \sigma_{\mathrm{II}}^{i}, \sigma_{\mathrm{III}}^{i}\right)$ the optimization problem

$$
\begin{aligned}
& \min \left(\sigma_{\mathrm{I}}-\sigma_{\mathrm{I}}^{i}\right)^{2}+\left(\sigma_{\mathrm{II}}-\sigma_{\mathrm{II}}^{i}\right)^{2}+\left(\sigma_{\mathrm{III}}-\sigma_{\mathrm{III}}^{i}\right)^{2} \\
& \text { subjected to } \Phi\left(\sigma_{\mathrm{I}}, \sigma_{\mathrm{II}}, \sigma_{\mathrm{III}}\right)=0
\end{aligned}
$$

must be solved. The solution is obtained using the LAGRANGE multiplier:

$$
F\left(\sigma_{\mathrm{I}}, \sigma_{\mathrm{II}}, \sigma_{\mathrm{III}}, \lambda\right)=\left(\sigma_{\mathrm{I}}-\sigma_{\mathrm{I}}^{i}\right)^{2}+\left(\sigma_{\mathrm{II}}-\sigma_{\mathrm{II}}^{i}\right)^{2}+\left(\sigma_{\mathrm{III}}-0\right)^{2}-\lambda \Phi\left(\sigma_{\mathrm{I}}, \sigma_{\mathrm{II}}, \sigma_{\mathrm{III}}\right)
$$

Its stationary points are the solutions of the equation $\nabla F=0$. The latter equation has multiple solutions, however only the point $\left(z_{\mathrm{I}}^{i}, z_{\mathrm{II}}^{i}, z_{\mathrm{III}}^{i}\right)$ on the failure surface, which has the minimal distance to the point $\left(\sigma_{\mathrm{I}}^{i}, \sigma_{\mathrm{II}}^{i}, \sigma_{\mathrm{III}}^{i}\right)$, is needed. Since the number of different solutions is small, the point of minimal distance can be found by trial and error. Finally, the value $f_{3 \mathrm{D}}$ of the estimation computes to

$$
f_{3 \mathrm{D}}:=\frac{1}{n-1} \sum_{i=1}^{n} \sqrt{\left(\sigma_{\mathrm{I}}^{i}-z_{\mathrm{I}}^{i}\right)^{2}+\left(\sigma_{\mathrm{II}}^{i}-z_{\mathrm{II}}^{i}\right)^{2}+\left(\sigma_{\mathrm{III}}^{i}-z_{\mathrm{III}}^{i}\right)^{2}} .
$$


ii) The second way to evaluate the quality of the approximation is to compute the minimal distance not in the principal stress space, but for a plane stress state (e.g. $\sigma_{\mathrm{III}}=0$ ). This criterion makes sense for special experimental data only. The optimization problem (6) is reduced to

$$
\begin{aligned}
& \min \left(\sigma_{\mathrm{I}}^{i}-\sigma_{\mathrm{I}}\right)^{2}+\left(\sigma_{\mathrm{II}}^{i}-\sigma_{\mathrm{II}}\right)^{2} \\
& \text { subjected to } \Phi\left(\sigma_{\mathrm{I}}, \sigma_{\mathrm{II}}, 0\right)=0 .
\end{aligned}
$$

Further proceed analogous to the case i). For each measurement $\left(\sigma_{\mathrm{I}}^{i}, \sigma_{\mathrm{II}}^{i}, 0\right)$ find the point of minimal distance $\left(z_{\mathrm{I}}^{i}, z_{\mathrm{II}}^{i}, 0\right)$ on the curve $\Phi\left(\sigma_{\mathrm{I}}, \sigma_{\mathrm{II}}, 0\right)=0$ and compute the value $f_{2 \mathrm{D}}$

$$
f_{2 \mathrm{D}}:=\frac{1}{n-1} \sum_{i=1}^{n} \sqrt{\left(\sigma_{\mathrm{I}}^{i}-z_{\mathrm{I}}^{i}\right)^{2}+\left(\sigma_{\mathrm{II}}^{i}-z_{\mathrm{II}}^{i}\right)^{2}} .
$$

iii) For a model built up upon the concept of equivalent stress, i. e.

$$
\Phi\left(\sigma_{\mathrm{I}}, \sigma_{\mathrm{II}}, \sigma_{\mathrm{III}}, \sigma_{\mathrm{eq}}\right)=0,
$$

with $\sigma_{\mathrm{eq}}=\sigma_{+}$, a simple evaluation criterion can be formulated. Whereas, for fitting of the model to the measurements, $\sigma_{\text {eq }}$ is considered to be a parameter. The value of $\sigma_{\text {eq }}$ which was obtained from fitting, is denoted by $\sigma_{\mathrm{eq}}^{*}$. In order to estimate the quality of approximation, for each measurement $\left(\sigma_{\mathrm{I}^{\prime}}^{i}, \sigma_{\mathrm{II}}^{i}, \sigma_{\mathrm{III}}^{i}\right), i=1 . . n$ the value $\sigma_{\mathrm{eq}}=\sigma_{\mathrm{eq}}^{i}$ should be computed with the property, that the respective measurement belongs to the surface $\Phi\left(\sigma_{\mathrm{I}}, \sigma_{\mathrm{II}}, \sigma_{\mathrm{III}}, \sigma_{\mathrm{eq}}^{i}\right)=0$. The equation

$$
\Phi\left(\sigma_{\mathrm{I}}^{i}, \sigma_{\mathrm{II}}^{i}, \sigma_{\mathrm{III}}^{i}, \sigma_{\mathrm{eq}}^{i}\right)=0
$$

must be solved with respect to $\sigma_{\text {eq }}^{i}$ for each $i=1 \ldots n$. The value of the estimation $f_{\text {eq }}$ computes to

$$
f_{\mathrm{eq}}:=\frac{1}{n-1} \sum_{i=1}^{n}\left|\frac{\sigma_{\mathrm{eq}}^{i}-\sigma_{\mathrm{eq}}^{*}}{\sigma_{\mathrm{eq}}^{*}}\right| .
$$

iv) Here the distance between the measurement and the surface of the model is measured along the straight line connecting the origin and the measurement, i. e. the measurement is compared to the respective stress state on the model surface (compression with compression, tension with tension etc.).

The criterion i) can be applied to any model and any set of measurements. However, it is rather often the case that the measurements are provided for a plane stress state, so the criterion ii) matches with the nature of the measurements. It is based on the assumption that the measurements, which belong to a plane stress state, should be approximated by points of the model, which also belong to a plane stress state. The criterion iii) can be applied to the models based on the concept of equivalent stress only. Further it can be expanded so that comparison can be performed for a distinguished stress state (e. g. torsion, compression, etc.). The criterion iv) is apparently physically sensible, however it is doubtful, if application of this criterion to an arbitrary stress state really makes sense. But for certain measurements (tension, compression, torsion etc.) it fits well.

The model (19) can be rewritten in the form $\Phi\left(I_{1}, I_{2}^{\prime}, I_{3}^{\prime}, \sigma_{\text {eq }}\right)=0$. For a set of measurements given in the principal stresses $\left(\sigma_{\mathrm{I}}^{i}, \sigma_{\mathrm{II}}^{i}, \sigma_{\mathrm{III}}^{i}\right), i=1$...n a simple objective function can be formulated:

$$
\mathrm{f}=\sum_{i=0}^{n}\left|\Phi\left(c_{3}, c_{6}, \gamma_{1}, \gamma_{2}, \gamma_{3}, \sigma_{\mathrm{I}}^{i}, \sigma_{\mathrm{II}}^{i}, \sigma_{\mathrm{III}}^{i}\right)\right|^{m}
$$

with $m=1,2$ or alternatively

$$
\mathrm{f}=\max _{i=1 \ldots n}\left|\Phi\left(c_{3}, c_{6}, \gamma_{1}, \gamma_{2}, \gamma_{3}, \sigma_{\mathrm{I}}^{i}, \sigma_{\mathrm{II}}^{i}, \sigma_{\mathrm{III}}^{i}\right)\right|
$$




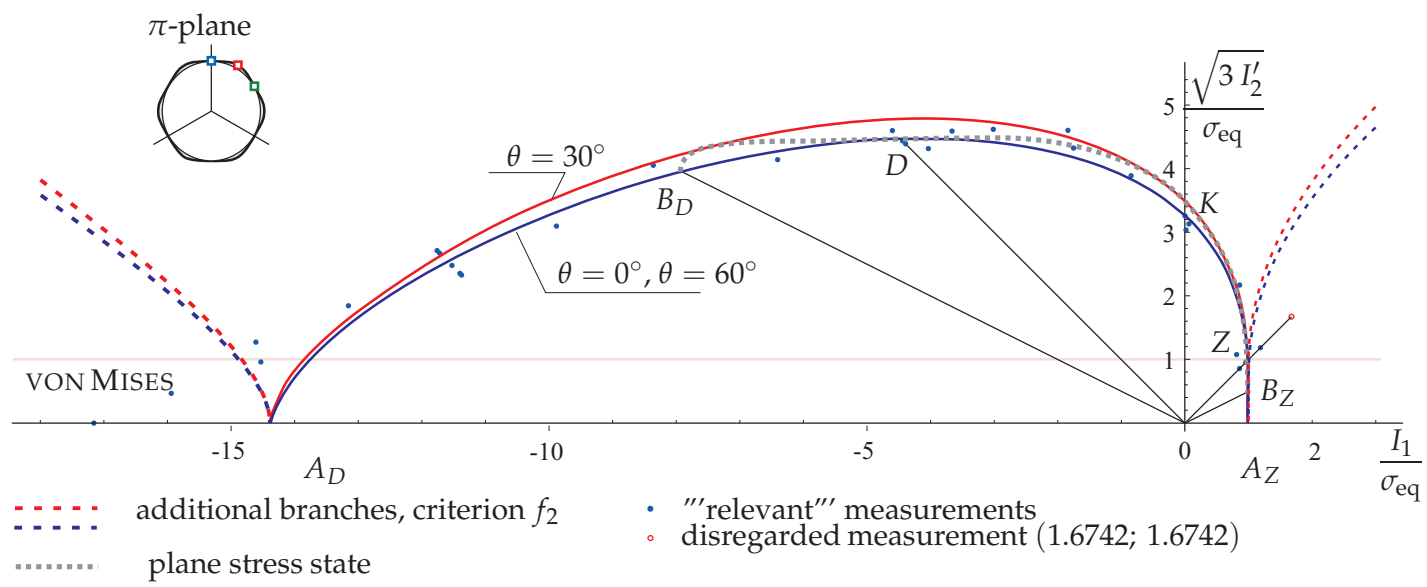

Fig. 10. Measurements by DERUNTZ in the BURZYŃSKI-plane fitted to the model Eq. (2):

$\mathrm{f} \rightarrow \min , n=2$ (Eq. 14) with parameters $\gamma_{1}=0.9753, \gamma_{2}=\gamma_{3}=-0.0695, c_{3}=0, c_{6}=1 / 2$ (measurement at tension with coordinates $\sigma(3220 / 1923 ; 0 ; 0)$ is disregarded)

Other values of $m$ are possible as well, but they are not helpful in applications. Now the following optimization problem can be formulated:

$$
\operatorname{minimize} f\left(c_{3}, c_{6}, \gamma_{1}, \gamma_{2}, \gamma_{3}\right)
$$

A solution leads to a model, which approximates the measurements $\left(\sigma_{\mathrm{I}}^{i}, \sigma_{\mathrm{II}}^{i}, \sigma_{\mathrm{III}}^{i}\right), i=1 \ldots n$. The optimization problem Eq. (16) is subjected to certain constraints.

\section{Analysis of Measured Data}

\subsection{Evaluation of Data from DE'RunTz-HoffMAN}

In [1] the limit behavior of syntactic foams is studied. Syntactic foam is a two-phase composite material with hollow microspheres integrally cast in a resin matrix. Glass spheres have a diameter $20 \ldots 80 \mu \mathrm{m}$ with wall thickness of $1 . . .3 \%$ of their diameters. A feature of the test is, that the hydrostatic tension is measured.

A reliable approximation is obtained with $c_{3}=0, c_{6}=1 / 2$ (Fig.10). In this case the meridians $\theta=0^{\circ}$ and $60^{\circ}$ coincide. The approximation of the 3D compression is thereby not accurate. The higher values of the $3 \mathrm{D}$ compression can be explained by the design of the experiment.

\subsection{Evaluation of Data from Despande-Fleck}

Two foams made of polyvinylchlorid (PVC) with different densities (H100 with $\rho=100 \mathrm{~kg} / \mathrm{m}^{3}$ and $\mathrm{H} 200 \rho=200 \mathrm{~kg} / \mathrm{m}^{3}$ ) were considered in [2]. The cell sizes are $s=400 \mu \mathrm{m}$ and $200 \mu \mathrm{m}$ respectively. The characteristical dimension of the specimen is taken to be $L \geq 50 \mathrm{~s}$. The measurements from [2] were read in using the tool TechDig 2.0d, normalized by the factor $\sigma_{+}=1.76 \mathrm{MPa}$ and represented in the BURZYŃSKI-plane (Fig. 11).

The measurements were approximated by the model (1). In order to obtain a continuously differentiable approximation the parameters $c_{3}\left(I_{1}\right)$ and $c_{6}\left(I_{1}\right)$ linearly depending on $I_{1}$ were taken (Fig. 8). The cross-section changes from the form with $(-1.1457 ; 0,2136)$ for $\gamma_{2}=-1 / 1,8$ to the rotationally symmetric form with $(0 ; 0)$ for $\gamma_{1}=1 / 3$. Application of such surfaces leads to higher computational complexity. 


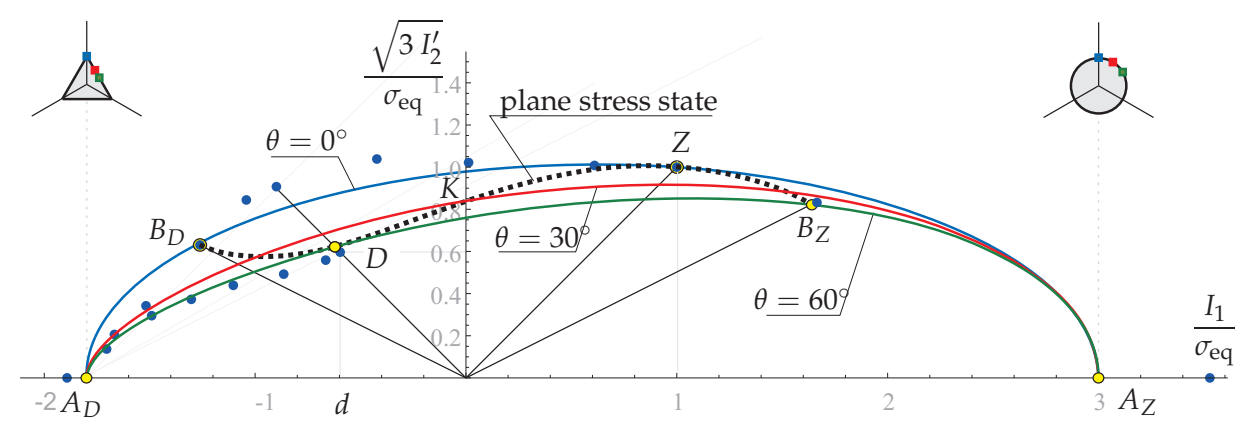

Fig. 11. Model with $c_{3}\left(I_{1}\right), c_{6}\left(I_{1}\right)$, Eq. (1), $\gamma_{1}=1 / 3, \gamma_{2}=-1 / 1,8$. The value $\left(\max I_{1}\right) / \sigma_{+}=3$ is taken from the Normal Stress Hypothesis. $Z$ - tension, $K$ - torsion, $D$ - compression, $B_{Z}$ - biaxial tension, $B_{D}$ - biaxial compression

\section{Summary}

In this paper six testing plans for the analysis of the limit behavior of hard foams are discussed. The models Eq. (1) and Eq. (2) are chosen for the approximation of the measurements. Models of closed surfaces for the description of the limit behavior are formulated. The parameters of the model $c_{3}, c_{6}, \gamma_{i}$ are computed using optimization routines based on objective functions implemented in MATHEMATICA 7. The optimization routine can be iterated in order to find the values $\left.\left.v_{+} \in\right]-1 ; 1 / 2\right]$. The second restriction $\left.\left.v_{-} \in\right]-1 ; 1 / 2\right]$ is a plausibility condition.

The identification of the limit surfaces Eq. (1) and Eq. (2) is carried out using the measurements from the literature [1,2]. For this kind of analysis the representation in the BURZYŃSKIplane can be used. In order to compare different optimization results the PARETO-analysis is used. Further approximations are based on measurements with PVC-foams (Airex with $\rho=40,100,150$ and $200 \mathrm{~kg} / \mathrm{m}^{3}$ ) and PMI-foams (Rohacell with $\rho=21,51,110$ and 200 $\left.\mathrm{kg} / \mathrm{m}^{3}\right)$.

The fitting routine described in the paper yields reliable results even for partially missing or uncertain measured data. In order to failure-safe structural members the models are implemented in FEM software ABAQUS.

\section{References}

1. J. A. DeRuntz, O. Hoffmann, J. of Appl. Mech., Transact. of the ASME, 36(9) (1969), 551 557.

2. V.S. Deshpande, N. A. Fleck, J. of the Mechanics and Physics of Solids, 48 (2000), 1253 1283.

3. V. A. Kolupaev, A. Bolchoun, H. Altenbach, 12th International Conference on Fracture ICF12, Proceedings, Ottawa, July 12-17, 2009, 10 p.

4. V. A. Kolupaev, A. Bolchoun, H. Altenbach, Konstruktion 5, (2009) pp. $59-66$

5. V. A. Kolupaev, A. Bolchoun, 12. Tagung Deformations- und Bruchverhalten von Kunststoffen, Book of Abstracts ed. by W. Grellmann, Merseburg (2009), pp. 73 - 87

6. V. A. Kolupaev, A. Bolchoun, Forschung im Ingenieurwesen, 72 5, (2008) pp. 209 - 232

7. V. A. Kolupaev, 3D-Creep Behaviour of Parts Made of Non-Reinforced Thermoplastics (in German), Martin-Luther Universität Halle-Wittenberg, Papierflieger Verlag, Clausthal- Zellerfeld, 2006 